\title{
Grid overlays reduce bias in mental representations of topographic maps
}

\author{
Lars Kuchinke $^{\mathrm{a},}$ *, Julian Keil ${ }^{\mathrm{b}}$, Dennis Edler ${ }^{\mathrm{b}}$, Anne-Kathrin Bestgen ${ }^{\mathrm{b}}$, Frank Dickmann ${ }^{\mathrm{b}}$ \\ ${ }^{a}$ Psychological Methods and Evaluation, IPU Berlin, Germany, lars.kuchinke@ipu-berlin.de \\ ${ }^{b}$ Cartography and Geo-Information Science, RUB Bochum, Germany \\ * Corresponding author
}

Keywords: Topographic Maps, Mental Representations, Spatial Cognition, Grids, Map Complexity, Object Location memory

\begin{abstract}
:
Reading spatial information from topographic maps to form mental representations that guide spatial orientation and navigation is a rather complex cognitive process. Perceptual and knowledge-driven processes interact to support the map reader in building these mental representations. The resulting cognitive maps are not one-to-one mappings of the spatial information and known to be distorted systematically. It is assumed that spatial information is hierarchically organized in these mental models. We are interested in how map design based on cognitive principles supports memory formation and leads to less distorted mental representations.

Based on the results of empirical studies we are able to show that overlaid grids in these maps address the hierarchical nature of these mental representations of map space. When map users are asked to learn object locations in a map the availability of overlaid grid layers improve object location memory. This effect is independent of the shape of these grid patterns (square grids or hexagonal grids) and, moreover, can be shown to be effective even in situations where the grids are interrupted by other maps layers (i.e. so-called illusory grids).

These results seem best explained by the formation of less distorted mental representations based on the availability of superordinate hierarchical information and the application of Gestalt principles by the map user. Thus again, point to the interaction between perceptual and knowledge-driven processes in the formation of these mental representations of map space. This assumption receives further support by eye-tracking data that reveal that grids do not only attract attention towards their own location but also seem to structure the gaze patterns in relation to the relevant object locations that are not necessarily located close to a grid line.
\end{abstract}

